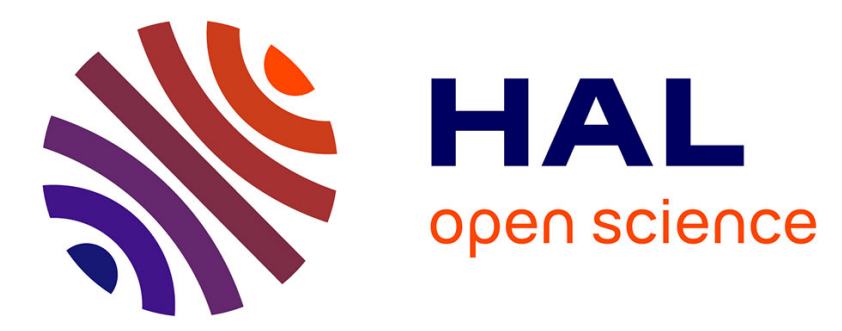

\title{
Implantation d'oxygène et co-implantation d'oxygène et de silicium, à faible dose, dans GaAs. I. Etude du phénomène de compensation
}

\author{
A. Le Bloa, Dang Tran Quan, Z. Guennouni, P.N. Favennec
}

\section{- To cite this version:}

A. Le Bloa, Dang Tran Quan, Z. Guennouni, P.N. Favennec. Implantation d'oxygène et co-implantation d'oxygène et de silicium, à faible dose, dans GaAs. I. Etude du phénomène de compensation. Revue de Physique Appliquée, 1989, 24 (10), pp.973-982. 10.1051/rphysap:019890024010097300 . jpa-00246135

\section{HAL Id: jpa-00246135 https://hal.science/jpa-00246135}

Submitted on 1 Jan 1989

HAL is a multi-disciplinary open access archive for the deposit and dissemination of scientific research documents, whether they are published or not. The documents may come from teaching and research institutions in France or abroad, or from public or private research centers.
L'archive ouverte pluridisciplinaire HAL, est destinée au dépôt et à la diffusion de documents scientifiques de niveau recherche, publiés ou non, émanant des établissements d'enseignement et de recherche français ou étrangers, des laboratoires publics ou privés. 


\title{
Implantation d'oxygène et co-implantation d'oxygène et de silicium, à faible dose, dans GaAs. I. Etude du phénomène de compensation
}

\author{
A. Le Bloa $\left({ }^{1}\right)$, Dang Tran Quan $\left({ }^{1}\right)$, Z. Guennouni $\left({ }^{1}\right)$ et P. N. Favennec $\left({ }^{2}\right)$ \\ (1) Groupe de Physique Cristalline, UA 804 CNRS, Université de Rennes I, Campus de Beaulieu, \\ 35042 Rennes Cedex, France \\ (2) Groupement ICM/TOH, Centre National d'Etudes des Télécommunications, Lannion B, 22301 Lannion \\ Cedex, France
}

(Reçu le 21 novembre 1988, révisé le 15 juin 1989, accepté le 30 juin 1989)

\begin{abstract}
Résumé. - Nous présentons les résultats de l'étude de la compensation des porteurs dans les échantillons de GaAs implantés ou co-implantés, à faible dose, en oxygène ou en oxygène et silicium. La compensation augmente avec la dose d'oxygène, s'étend bien au-delà de la région implantée et est nettement plus importante dans le cas d'une co-implantation " $\mathrm{Si}+\mathrm{O}$ » suivie d'un recuit à $650^{\circ} \mathrm{C}$. Le nombre d'électrons compensés est plus grand que celui des ions d'oxygène implanté. Celui-ci n'est pas seul responsable de la compensation. Cette compensation semble être due à la formation de nouveaux centres et aux modifications, avec piégeage de porteurs, des équilibres physico-chimiques entre les centres préexistants et entre les défauts complexes du réseau cristallin et les impuretés résiduelles du substrat par suite des implantations de l'oxygène et du silicium.

Abstract. - Compensation results in low dose oxygen-implanted and $\mathrm{Si}+\mathrm{O}$ coimplanted $\mathrm{GaAs}$ are presented. The compensation increases with oxygen dose, extends beyond the implantation limit and is more important in $\mathrm{Si}+\mathrm{O}$ coimplanted $650^{\circ} \mathrm{C}$ annealed samples. The number of compensated carrier is greater than the one of implanted oxygen. The latter is not responsable alone for the compensation effect. The compensation seems to be due first, to new deep levels created after implantations, second, to the modifications of physico-chemical equilibrium among deep levels and between cristallizing imperfections and residual impurities in GaAs substrate.
\end{abstract}

\section{Introduction.}

L'influence de l'oxygène, présent dans l'enceinte de préparation de $\mathrm{GaAs}$, sur les propriétés électriques de ce semiconducteur a été reconnue depuis environ une trentaine 'añn es ; en e et, on a cons a e que la résistivité du GaAs préparé dans ces conditions est très élevée. L'exploitation de ce résultat pour tenter de préparer du GaAs semi-isolant a fait l'objet de nombreux travaux de recherches. Ainslie et al. [2] ont utilisé de l'oxygène gazeux ; Fertin et al. [3], Woodall et Wood [4], Woodall [5], Martin et al. [6] ont introduit de l'oxygène sous forme de $\mathrm{Ga}_{2} \mathrm{O}_{3}$ lors de la préparation de $\mathrm{GaAs}$ par la méthode de Bridgman. Favennec et al. $[7,8]$, ont obtenu par implantation d'oxygène, une diminution importante du nombre d'électrons libres dans la couche implantée de GaAs. Les résultats obtenus par ces auteurs ont permis d'envisager la réalisation de circuits intégrés à partir de GaAs semi-isolant ainsi préparé. Cependant, si la technique de préparation de GaAs semi-isolant par l'adjonction d'oxygène aux e ements e syn ese peu e re consi e comme maîtrisée, il n'en est pas de même quant à la compréhension du mécanisme de la compensation produite par cette adjonction. L'hypothèse selon laquelle la compensation obtenue résulte d'une diminution des impuretés silicium par l'oxygène est admise par Ainslie et al. [2] ; Martin et al. [6] ont montré que la présence d'oxygène lors de la préparation de GaAs par la méthode Bridgman a permis de réduire la quantité de silicium dans le lingot obtenu par rapport à celle présente dans les produits initiaux de synthèse. Weiner et al. [9] ont proposé la forma- 
tion de complexes Si-O dans GaAs pour expliquer l'augmentation de la résistivité. Dans le but de mieux étudier les effets d'une telle interaction " oxygène-silicium », nous avons effectué l'étude de la compensation (Partie I) et des défauts profonds (Partie II) dans des échantillons de GaAs co-implantés en oxygène et en silicium. Nous donnons dans cet article les résultats concernant la compensation.

Nous décrirons d'abors les caractéristiques de nos échantillons et la procédure de leur caractérisation avant d'exposer les résultats expérimentaux et leur discussion.

\section{Caractéristique des échantillons.}

Pour effectuer notre étude, nous avons choisi un substrat de GaAs (Bridgman) (100) de type n, non intentionnellement dopé. La concentration électronique moyenne $y$ est de $1,4 \times 10^{16} \mathrm{~cm}^{-3}$. Ce substrat est multi-implanté en oxygène et implanté en silicium. Les conditions des multi-implantations d'oxygène ont été choisies pour que la répartition calculée de l'oxygène soit sensiblement linéaire dans une région comprise entre 0,25 et $0,75 \mu \mathrm{m}$ de profondeur (Fig. 1). Une telle répartition est obte- nue par trois implantations d'énergie et de dose bien déterminées que nous préciserons par la suite. Cette répartition linéaire de l'oxygène avait pour objet de mieux comparer les concentrations de défauts déterminés expérimentalement à la concentration calculée de l'oxygène et ainsi de mieux reconnaître les défauts corrélés à la présence de celui-ci. D'autre part, le gradient de la concentration d'oxygène est alors plus faible que celui existant dans le cas d'une mono-implantation. On peut alors espérer que la variation de la concentration électronique en fonction de la profondeur sera plus graduelle et que sa détermination à partir d'une caractéristique $C(V)$ ( $C$ et $V$ sont respectivement la capacité et la tension de polarisation de la diode de mesure) sera alors plus proche de la concentration globale $N_{\mathrm{D}}-N_{\mathrm{A}}\left(N_{\mathrm{A}}\right.$ : concentration résiduelle des accepteurs et $N_{\mathrm{D}}$ : concentration des donneurs).

Une première multi-implantation d'oxygène que nous noterons $\left(\Phi_{\mathrm{O}}\right)$ consiste en trois implantations successives dont les énergies et les doses des ions sont données dans le tableau I.

Une deuxième multi-implantation d'oxygène notée $\left(2 \Phi_{\mathrm{O}}\right)$ consiste en trois implantations aux mêmes énergies que pour $\Phi_{O}$ mais avec une dose double pour chacune des trois implantations.

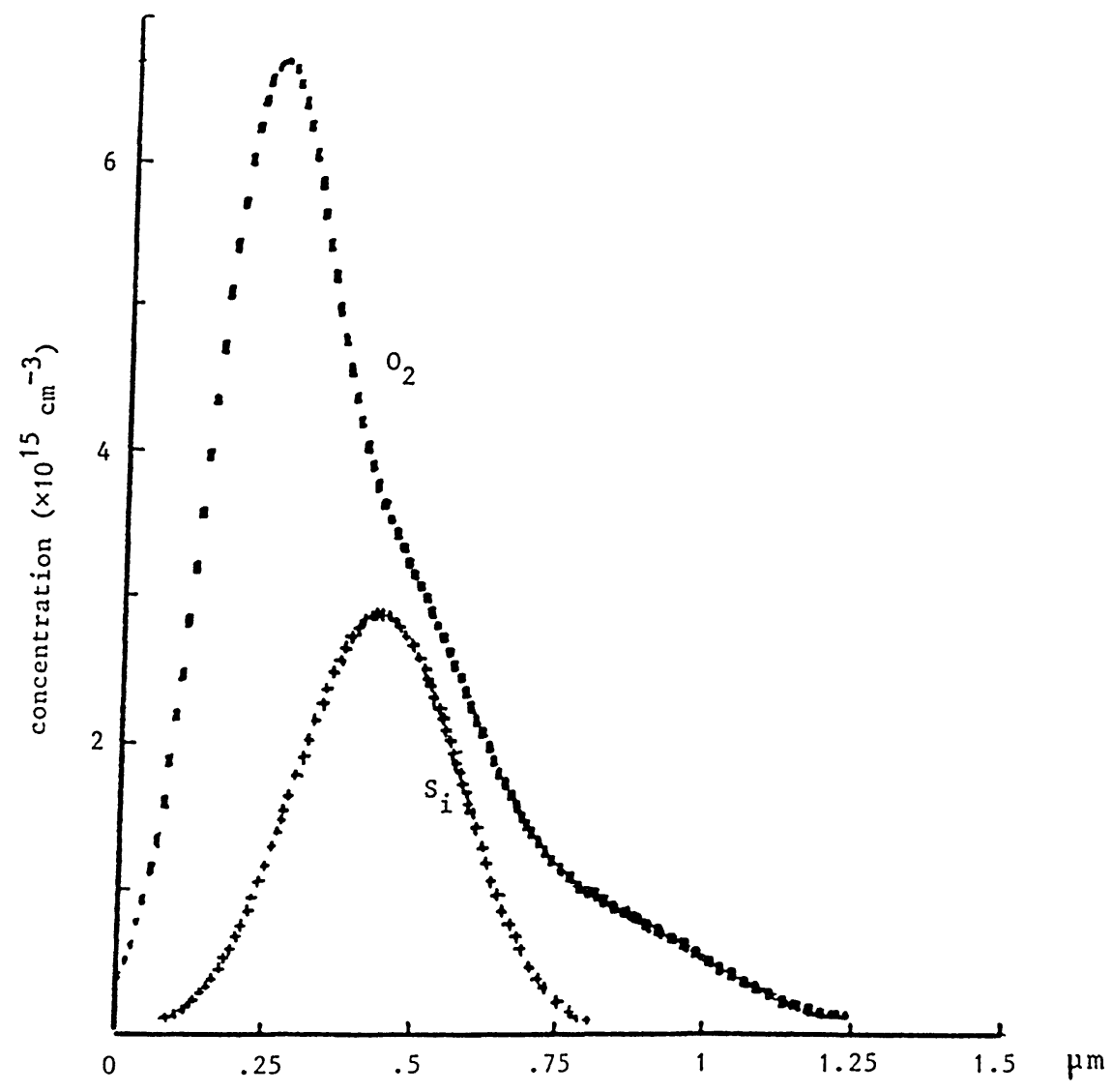

Fig. 1. - Concentrations calculées d'ions oxygène et silicium implantés en fonction de la profondeur. (Cas des doses simples : $\Phi_{\mathrm{O}}$ et $\left.\Phi_{\mathrm{Si}}\right)$.

[Calculated concentration depth profiles of oxygen and silicon ions implanted (single doses $\Phi_{\mathrm{O}}, \Phi_{\mathrm{Si}}$ ) into GaAs.] 
Tableau I. - Doses et énergies des trois implantations composant la première multi-implantation notée $\Phi_{\mathrm{O}}$. La dose et l'énergie de la première implantation de silicium $\left(\Phi_{\mathrm{Si}}\right)$ sont aussi indiquées.

[Doses and energies of oxygen multi-implantation $\left(\Phi_{\mathrm{O}}\right)$ and silicon implantation $\left(\Phi_{\mathrm{Si}}\right)$ into GaAs.]

\begin{tabular}{|l|c|c|c|c|}
\hline & \multicolumn{3}{|c|}{ Oxygène $\left(\Phi_{\mathrm{O}}\right)$} & Silicium $\left(\Phi_{\mathrm{Si}}\right)$ \\
\hline dose $\left(\mathrm{cm}^{-2}\right)$ & $1,4 \times 10^{11}$ & $1,05 \times 10^{11}$ & $4,2 \times 10^{10}$ & $10^{11}$ \\
\hline énergie $(\mathrm{keV})$ & 150 & 290 & 550 & 500 \\
\hline
\end{tabular}

Les deux multi-implantations d'oxygène ont été choisies pour que la concentration maximale calculée de l'oxygène soit :

- inférieure à la concentration électronique du substrat pour $\Phi_{\mathrm{O}}$;

- voisine de cette concentration pour $2 \Phi_{0}$.

Dans certains échantillons, à la première multiimplantation d'oxygène $\Phi_{\mathrm{O}}$ dans GaAs on superpose une implantation de silicium $\Phi_{\mathrm{Si}}$ dont l'énergie et la dose sont indiquées dans le tableau I. De même, à la deuxième multi-implantation d'oxygène dans un échantillon, on fait suivre une implantation de silicium $2 \Phi_{\mathrm{Si}}$ de même énergie que $\Phi_{\mathrm{Si}}$ mais de dose double. La première et la deuxième co-implantation sont notées respectivement $\left(\Phi_{\mathrm{O}}, \Phi_{\mathrm{Si}}\right)$ et $\left(2 \Phi_{\mathrm{O}}\right.$, $\left.2 \Phi_{\mathrm{Si}}\right)$. La dose de silicium a été choisie pour que la concentration maximale calculée de cet élément soit sensiblement voisine de la concentration calculée de l'oxygène à la profondeur de ce maximum (Fig. 1) $\left(3,5 \times 10^{15} \mathrm{~cm}^{-3}\right)$. Nous avons ainsi, dans le même échantillon, une région où la concentration d'oxygène est plus forte que celle du silicium, et une autre où ces concentrations sont du même ordre de grandeur.
Tous les échantillons implantés ont été recuits, après encapsulation sous $\mathrm{Si}_{3} \mathrm{~N}_{4}$, soit à $650{ }^{\circ} \mathrm{C}$, soit à $900{ }^{\circ} \mathrm{C}$, pendant $15 \mathrm{~min}$. Les couches de $\mathrm{Si}_{3} \mathrm{~N}_{4}$ sont produites par pulvérisation cathodique réactive. Trois échantillons non implantés $N^{\circ} 1,2$ et 3 nous servent de référence. Seuls les échantillons $\mathrm{N}^{\circ} 2$ et 3 ont été recuits respectivement à $650^{\circ} \mathrm{C}$ et $900{ }^{\circ} \mathrm{C}$ pendant $15 \mathrm{~min}$ comme les échantillons implantés.

Dans la suite nous repérerons chaque diode par son numéro, suivi des indications de son type d'implantation en oxygène puis en silicium et de la température de recuit. La non-existence d'un traitement sera exprimée par un astérisque : par exemple, $\mathrm{N}^{\circ} 1:(*, *, *)$ échantillon ni implanté ni recuit ; $\mathrm{N}^{\circ} 4:\left(\Phi_{\mathrm{O}},{ }^{*}, 650\right)$ échantillon multi-implanté en oxygène $\left(\Phi_{\mathrm{O}}\right)$, non implanté en silicium, recuit à $650^{\circ} \mathrm{C}$. (Le Tab. II donne les caractéristiques des échantillons étudiés).

\section{Caractérisation.}

Les mesures ont été effectuées sur des diodes Schottky faites sur des échantillons de GaAs que nous avons préparés en utilisant des contacts en or.

Tableau II. - Caractéristiques des échantillons: $\Phi_{\mathrm{O}}$ et $2 \Phi_{\mathrm{O}}$ sont les multi-implantations d'oxygène (les implantations de $2 \Phi_{\mathrm{O}}$ sont effectuées aux mêmes énergies mais à des doses doubles de celles de $\left.\Phi_{\mathrm{O}}\right), \Phi_{\mathrm{Si}}$ et $2 \Phi_{\mathrm{Si}}$ sont les implantations de silicium $\left(2 \Phi_{\mathrm{Si}}\right.$ est une implantation à la même énergie mais à une dose double de celle de $\Phi_{\mathrm{Si}}$. Les énergies et les doses de $\Phi_{\mathrm{O}}$ et $\Phi_{\mathrm{Si}}$ sont données dans le tableau I. Le substrat non dopé de GaAs est de type n avec une concentration électronique de $1,4 \times 10^{16} \mathrm{~cm}^{-3}$. La durée des deux recuits est de $15 \mathrm{~min}$; l'astérisque indique qu'il n'y a pas d'implantation ou de recuit.

[Characteristics of GaAs samples: $\Phi_{\mathrm{O}}$ and $2 \Phi_{\mathrm{O}}$ : oxygen multi-implantation, single and double dose ; $\Phi_{\mathrm{Si}}$ and $2 \Phi_{\mathrm{Si}}$ : Si implantation, single and double dose. $\left({ }^{*}\right):$ no implantation or no annealing. Annealing conditions : $650{ }^{\circ} \mathrm{C}$ or $900{ }^{\circ} \mathrm{C}$ for $15 \mathrm{~min}$.]

\begin{tabular}{|l|c|c|c|c|c|c|c|c|c|}
\hline \multicolumn{1}{|c|}{ Numéro des échantillons } & 1 & 2 & 3 & 4 & 5 & 6 & 7 & 8 & 9 \\
\hline Multi-implantation d'oxygène & $*$ & $*$ & $*$ & $\Phi_{\mathrm{O}}$ & $2 \Phi_{\mathrm{O}}$ & $\Phi_{\mathrm{O}}$ & $2 \Phi_{\mathrm{O}}$ & $\Phi_{\mathrm{O}}$ & $2 \Phi_{\mathrm{O}}$ \\
\hline Implantation de silicium & $*$ & $*$ & $*$ & $*$ & $*$ & $\Phi_{\mathrm{Si}}$ & $*$ & $\Phi_{\mathrm{Si}}$ & $2 \Phi_{\mathrm{Si}}$ \\
\hline Température de recuit $\left({ }^{\circ} \mathrm{C}\right)$ & $*$ & 650 & 900 & 650 & 650 & 650 & 900 & 900 & 900 \\
\hline
\end{tabular}


Le contact ohmique est réalisé par le dépôt d'une couche mince d'alliage $\mathrm{Au}-\mathrm{Ge}$ suivi d'un recuit à $360^{\circ} \mathrm{C}$. Nous enregistrons d'abord le spectre DDLTS (Double Level Transient Spectroscopy) pour caractériser les défauts profonds [10]. Pour cela nous avons appliqué la méthode DDLTS utilisant une détection synchrone que nous avons mise au point [11]. Les variations de la concentration électronique en fonction de la profondeur ont été déterminées à partir d'un enregistrement de la caractéristique $C(V)$ à la température du maximum de chacun des pics du signal DDLTS. ( $C$ et $V$ représentent respectivement la capacité et la tension de polarisation de la diode). Cette concentration est en effet nécessaire pour déterminer celle des défauts (Partie II).

Pour cet enregistrement le pas de tension $\Delta V$ entre deux mesures successives est constant. La concentration électronique $n(W)$ en fonction de la profondeur $W$ est alors déduite des relations suivantes :

$$
W=\frac{\varepsilon_{\mathrm{s}} S}{C} ; n(W)=\frac{2}{q \varepsilon_{\mathrm{s}} S^{2}}\left[\frac{\mathrm{d}\left(C^{-2}\right)}{\mathrm{d} V}\right]^{-1}
$$

$\varepsilon_{\mathrm{s}}$ est la constante diélectrique du semiconducteur, $S$ l'aire de la diode et $q$ la charge électrique élémentaire.

Pour calculer $\left[\frac{\mathrm{d}\left(C^{-2}\right)}{\mathrm{d} V}\right]$, nous avons mis au point un programme pour faire une approximation polynomiale (utilisation de polynômes pseudoorthogonaux), au sens des moindres carrés, d'une fonction dont les valeurs sont connues à des points discrets.

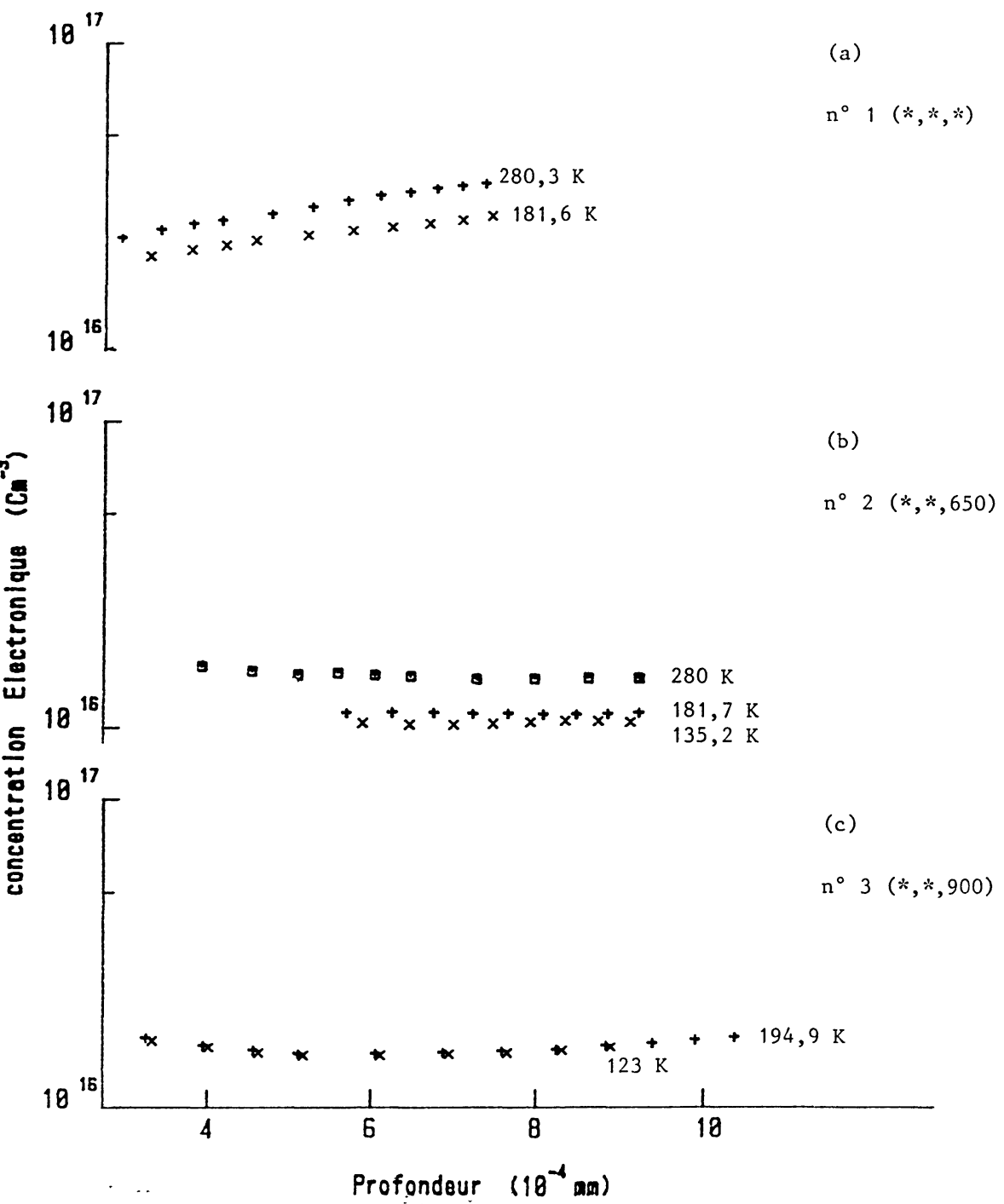

Fig. 2. - Concentration électronique des échantillons de référence non implantés. a : non recuit ; b : recuit à $650{ }^{\circ} \mathrm{C}$ pendant $15 \mathrm{~min}$; c : recuit à $900^{\circ} \mathrm{C}$ pendant $15 \mathrm{~min}$.

[Electron concentration depth profiles in unimplanted GaAs. a : unannealed GaAs ; b : annealed $\left(650^{\circ} \mathrm{C}, 15 \mathrm{~min}\right)$ GaAs ; c : annealed $\left.\left(900^{\circ} \mathrm{C}, 15 \mathrm{~min}\right) \mathrm{GaAs}.\right]$ 


\section{Résultats expérimentaux et leurs discussions.}

ECHANTILLONS NON IMPLANTÉS. - L'échantillon $\mathrm{N}^{\circ} 1\left({ }^{*},{ }^{*},{ }^{*}\right)$ a une concentration électronique qui augmente quasi linéairement avec la profondeur tandis que celle des échantillons $\mathrm{N}^{\circ} 2\left({ }^{*}, *, 650\right)$ et $\mathrm{N}^{\circ} 3(*, *, 900)$ en est indépendante. Dans les échantillons $\mathrm{N}^{\circ} 1$ et 2 cette concentration dépend aussi de la température de mesure (Figs. 2a et $2 \mathrm{~b}$ ). Elle est égale à celle donnée par le fabricant $\left(1,4 \times 10^{16} \mathrm{~cm}^{-3}\right)$ dans l'échantillon $\mathrm{N}^{\circ} 2$ seulement à $280,3 \mathrm{~K}$ et dans l'échantillon $\mathrm{N}^{\circ} 3$ aux deux températures de mesure. Nous en déduisons qu'un recuit à $900^{\circ} \mathrm{C}$ pendant $15 \mathrm{~min}$ est nécessaire pour obtenir une bonne homogénéité du substrat.

\section{ECHANTILLONS IMPLANTÉS.}

Echantillons $N^{\circ} 4$ et $N^{\circ} 5$. - La concentration électronique de l'échantillon $\mathrm{N}^{\circ} 4:\left(\Phi_{\mathrm{O}},{ }^{*}, 650\right)$ aug-

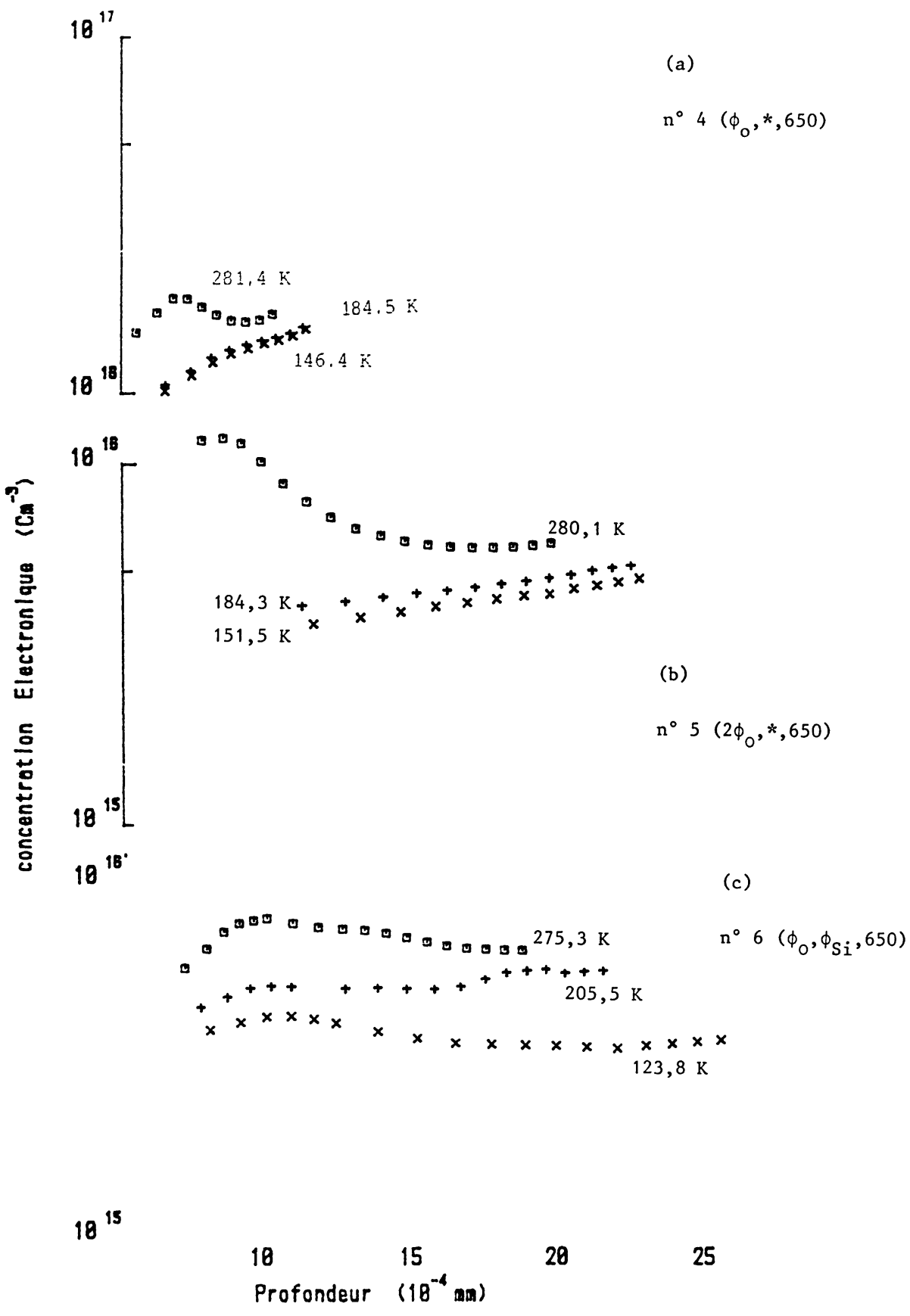

Fig. 3. - Concentration électronique des échantillons de GaAs implantés et recuits à $650^{\circ} \mathrm{C}$ pendant 15 min. a : implantés en oxygène ; $b$ : implantés en oxygène à double dose ; $c$ : co-implantés en oxygène et en silicium. [Electron concentration depth profiles in implanted $\mathrm{GaAs}$, after $\left(650^{\circ} \mathrm{C}, 15 \mathrm{~min}\right)$ annealing. a : oxygen implanted GaAs ; b : oxygen (double dose) implanted GaAs ; $\mathrm{c}$ : oxygen and silicon co-implanted GaAs).] 
mente avec la profondeur aux températures de $146,4 \mathrm{~K}$ et $184,5 \mathrm{~K}$, mais présente un faible maximum vers $0,75 \mu \mathrm{m}$ à la température de $281,4 \mathrm{~K}$ (Fig. 3a). Elle reste cependant voisine de celle du substrat : l'implantation d'oxygène dans ce cas ne produit pratiquement pas de compensation. La dose d'oxygène implanté est probablement trop faible ; en effet, le maximum calculé $\left(7 \times 10^{15} \mathrm{~cm}^{-3}\right)$ (Fig. 1) de la concentration d'oxygène est voisin de la concentration d'oxygène résiduel déterminée par Shikano et al. [12] dans les lingots de GaAs préparés par la méthode Bridgman. La compensation augmente avec la dose d'oxygène implanté. Dans l'échantillon $\mathrm{N}^{\circ} 5\left(2 \Phi_{\mathrm{O}}, *, 650\right)$ qui a reçu une double dose d'oxygène, cette concentration est inférieure à $5 \times 10^{15} \mathrm{~cm}^{-3}$ à $1,5 \mu \mathrm{m}$ (Fig. $3 b$ ).

Echantillon $N^{\circ}$ 6. - La concentration électronique de l'échantillon $\mathrm{N}^{\circ} 6:\left(\Phi_{\mathrm{O}}, \Phi_{\mathrm{Si}}, 650\right)$ dépend également de la température; ses variations en fonction de la profondeur présentent un maximum au voisinage de $1 \mu \mathrm{m}$ aux trois températures de détermination (Fig. 3c) ; ce maximum est d'autant plus net que la température de mesure est plus grande ; c'est une profondeur supérieure à celle correspondant au maximum de la concentration calculée du silicium implanté (environ $0,4 \mu \mathrm{m}$ ). A la température de $205,5 \mathrm{~K}$ et au voisinage de $1,5 \mu \mathrm{m}$, la concentration électronique augmente légèrement avec la profondeur, tandis qu'à $275,3 \mathrm{~K}$, entre 1 et $1,5 \mu \mathrm{m}$ c'est une diminution de la concentration électronique qui se produit. L'augmentation de la concentration, aux environs de $1,5 \mu \mathrm{m}$, à $205,5 \mathrm{~K}$, peut être due à l'ionisation d'un défaut non encore ionisé aux températures plus basses. La concentration de ce défaut déduite de la concentration électronique est au moins égale à $0,5 \times 10^{15} \mathrm{~cm}^{-3}$. L'augmentation de la concentration électronique en fonction de la température (entre $124 \mathrm{~K}$ et $276 \mathrm{~K}$ ) est due à l'ionisation de plusieurs défauts lorsque la température croît.

On peut déduire l'ordre de grandeur de l'énergie d'ionisation $E_{\mathrm{C}}-E_{\mathrm{t}}\left(E_{\mathrm{t}}\right.$ niveau d'énergie du défaut) à partir de la position du niveau de Fermi $E_{\mathrm{F}}$. En effet, l'ensemble des défauts considérés est à moitié rempli d'électrons lorsque $E_{\mathrm{t}}=E_{\mathrm{F}} . E_{\mathrm{F}}$ est donné par :

$$
E_{\mathrm{C}}-E_{\mathrm{F}}=k T \ln \left(\frac{N_{\mathrm{C}}}{n}\right)
$$

où $E_{\mathrm{C}}$ est le niveau d'énergie du bas de la bande de conduction, $N_{\mathrm{C}}$ la densité effective des états de cette bande et $n$ la concentration électronique.

Le tableau III donne les valeurs des énergies d'ionisation thermique $\left(E_{\mathrm{C}}-E_{\mathrm{t}}\right)$ déterminées aux trois températures $151 \mathrm{~K}, 184 \mathrm{~K}$ et $280 \mathrm{~K}$ pour l'échantillon $\mathrm{N}^{\circ} 5$, et $123,8 \mathrm{~K}, 205,5 \mathrm{~K}$ et $275,3 \mathrm{~K}$ pour l'échantillon $\mathrm{N}^{\circ} 6$, en utilisant les valeurs de la concentration électronique correspondant respectivement à une profondeur de $1,2 \mu \mathrm{m}$ (pour l'échantillon $\mathrm{N}^{\circ} 5$ ) et de 1,7 $\mu \mathrm{m}$ (pour l'échantillon $\mathrm{N}^{\circ} 6$ ).

Ce tableau montre que l'énergie d'ionisation (ou les énergies d'ionisation si les centres présents ont plusieurs niveaux d'énergie) est (sont) de l'ordre de grandeur de 0,05 à $0,1 \mathrm{eV}$. Abdalla et al. [13] ont mis en évidence une énergie d'activation de $0,04 \mathrm{eV}$ de la conductivité d'une couche de GaAs implantée en oxygène (concentration sensiblement constante) recuite à $470{ }^{\circ} \mathrm{C}$ pendant $30 \mathrm{~min}$. D'autre part, nous avons mis en évidence (deuxième partie) dans cet échantillon $\mathrm{N}^{\circ} 6$ un défaut dont le taux d'émission électronique a une énergie d'activation de $0,11 \mathrm{eV}$. Ce défaut est probablement l'un de ceux qui produisent les modifications de la concentration électronique que nous observons.

Comparaison entre les concentrations électroniques des échantillons $N^{\circ} 4,5$, et 6 , recuits à $650^{\circ} \mathrm{C}$. Considérons les échantillons $\mathrm{N}^{\circ} 4\left(\Phi_{\mathrm{O}},{ }^{*}, 650\right)$ et $\mathrm{N}^{\circ} 6\left(\Phi_{\mathrm{O}}, \Phi_{\mathrm{Si}}, 650\right)$ qui ne diffèrent l'un de l'autre que par la présence de silicium co-implanté (Figs. 3a et 3c). A une profondeur donnée et quelle que soit la température de mesure, la concentration électronique de l'échantillon $\mathrm{N}^{\circ} 4$ est plus élevée et varie moins avec la température que celle de l'échantillon $\mathrm{N}^{\circ} 6$. A cette température de recuit de $650^{\circ} \mathrm{C}$, la présence de silicium co-implanté produit une compensation plus importante de GaAs que dans le cas d'une seule multi-implantation d'oxygène.

Tableau III. - Valeurs de $E_{\mathrm{C}}-E_{\mathrm{F}}$ : positions du niveau de Fermi $E_{\mathrm{F}}$ par rapport au bas de la bande de conduction $E_{\mathrm{C}}$, aux trois températures de détermination de la concentration électronique, aux profondeurs de $1,2 \mu \mathrm{m}$ (échantillon $N^{\circ} 5:\left(2 \Phi_{\mathrm{O}},{ }^{*}, 650\right)$ ) et $1,7 \mu \mathrm{m}$ (échantillon $\left.N^{\circ} 6:\left(\Phi_{\mathrm{O}}, \Phi_{\mathrm{Si}}, 650\right)\right)$.

[Thermal ionisation energy at different temperatures deduced from electronic concentrations at $1.2 \mu \mathrm{m}$ (for sample $\left.\mathrm{N}^{\circ} 5\left(2 \Phi_{\mathrm{O}}, *, 650\right)\right)$ and at $1.7 \mu \mathrm{m}$ (for sample $\mathrm{N}^{\circ} 6\left(\Phi_{\mathrm{O}}, \Phi_{\mathrm{Si}}, 650\right)$ ).

\begin{tabular}{|l|c|c|c|c|c|c|}
\hline \multicolumn{1}{|c|}{ Echantillon } & \multicolumn{3}{|c|}{5} & \multicolumn{3}{c|}{6} \\
\hline Température (K) & 151 & 184 & 280 & 123,8 & 205,5 & 275,3 \\
Concentration électronique & $3 \times 10^{15}$ & $4,3 \times 10^{15}$ & $10^{16}$ & $3 \times 10^{15}$ & $5,5 \times 10^{15}$ & $6,5 \times 10^{15}$ \\
$\left(\mathrm{~cm}^{-3}\right)$ & 0,049 & 0,061 & 0,092 & 0,058 & 0,0684 & 0,102 \\
$E_{\mathrm{C}}-E_{\mathrm{F}}(\mathrm{eV})$ & & & & & & \\
\hline
\end{tabular}


Echantillons $N^{\circ} 7,8$ et 9 recuits à $900{ }^{\circ} \mathrm{C}$. - Les résultats obtenus après ce recuit sont assez différents de ceux obtenus après le recuit à $650^{\circ} \mathrm{C}$. Dans l'échantillon $\mathrm{N}^{\circ} 7$ : $\left(2 \Phi_{\mathrm{O}},{ }^{*}, 900\right)$, la compensation des donneurs s'étend au-delà de 1,5 $\mu \mathrm{m}$ (Fig. 4a). Dans le cas de l'échantillon $\mathrm{N}^{\circ} 8\left(\Phi_{\mathrm{O}}, \Phi_{\mathrm{Si}}, 900\right)$ la concentration électronique est importante au voisinage de la surface ; elle diminue quand la profondeur augmente. Zohta [16] a aussi observé ce comportement dans des échantillons de GaAs non dopé après un recuit sans encapsulation de $\mathrm{Si}_{3} \mathrm{~N}_{4}$ à $860{ }^{\circ} \mathrm{C}$; il attribue ce phénomène à une émission d'électrons par les défauts présents au voisinage de la surface.
L'échantillon $\mathrm{N}^{\circ} 9$ : $\left(2 \Phi_{\mathrm{O}}, 2 \Phi_{\mathrm{Si}}, 900\right)$ présente aux profondeurs de 0,6 à $1,5 \mu \mathrm{m}$, une concentration électronique plus élevée que celle de l'échantillon $\mathrm{N}^{\circ} 7$ : $\left(2 \Phi_{\mathrm{O}},{ }^{*}, 900\right)$. La comparaison des variations des concentrations électroniques en fonction de la profondeur pour les deux échantillons précédents (Figs. 4a et 4c) montre qu'entre les profondeurs de 0,6 et $1,5 \mu \mathrm{m}$, le nombre d'électrons supplémentaires dans l'échantillon $\mathrm{N}^{\circ} 9$ est grossièrement de l'ordre de grandeur du nombre d'ions $\mathrm{Si}$ implantés. On notera également que les valeurs élevées de la concentration de l'échantillon $\mathrm{N}^{\circ} 9$ s'étendent à des profondeurs (de 0,6 à $1,5 \mu \mathrm{m}$ ) supérieures à celles
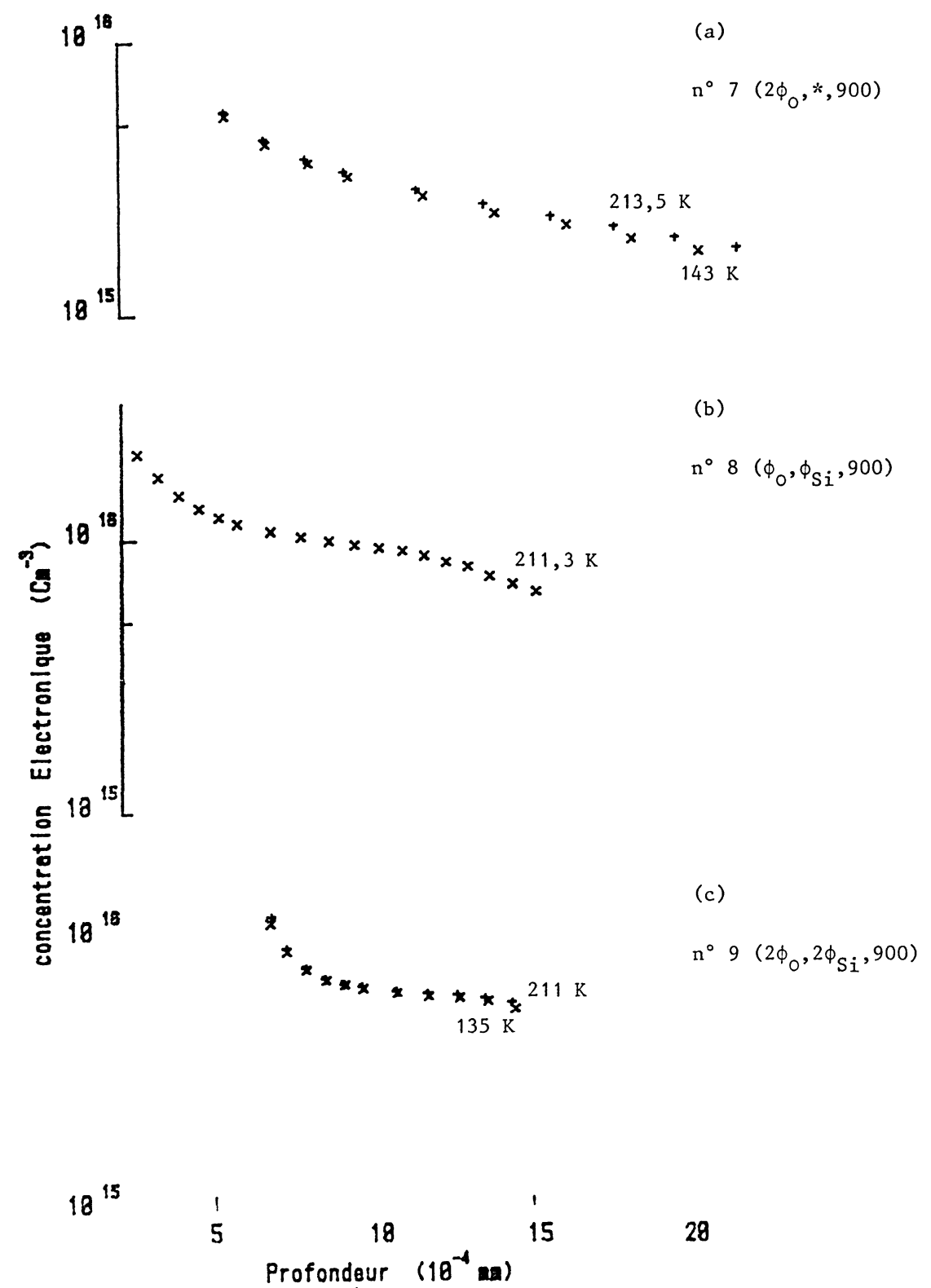

Fig. 4. - Concentration électronique des échantillons de GaAs implantés et recuits à $900{ }^{\circ} \mathrm{C}$ pendant 15 min. a : implantés en oxygène ; $b$ : co-implantés en oxygène et en silicium ; $c$ : co-implantés en oxygène et en silicium à double dose.

[Electron concentration depth profiles in implanted $\mathrm{GaAs}$, after $\left(900{ }^{\circ} \mathrm{C}, 15 \mathrm{~min}\right)$ annealing. a : oxygen implanted GaAs; b : oxygen and silicon co-implanted GaAs; c : oxygen and silicon (double dose) co-implanted GaAs).] 
calculées théoriquement pour l'implantation silicium $(0,25-0,75 \mu \mathrm{m}$; Fig. 1). Yeo et al. [17] ont obtenu des résultats semblables pour une implantation de $\mathrm{Si}$ dans GaAs (dose : $1,6 \times 10^{12} \mathrm{~cm}^{-2}$; énergie : $100 \mathrm{keV}$ et recuit à $900^{\circ} \mathrm{C}$ ).

Les résultats obtenus semblent montrer que le silicium implanté se comporte en donneur.

DiscUSSIONS. - La compensation produite par l'implantation d'oxygène est un phénomène complexe. Pour tenter de comprendre son mécanisme nous allons examiner l'influence des impuretés résiduelles du type « accepteurs » et des défauts du réseau cristallin de $\mathrm{GaAs}$ créés par l'implantation.

Le chrome est l'une de ces impuretés. Des mesures de SIMS, effectuées sur un substrat contenant du chrome $\left(4 \times 10^{15} \mathrm{~cm}^{-3}\right)$, implanté en oxygène (énergie : $1 \mathrm{meV}$; dose : $10^{14} \mathrm{~cm}^{-2}$ ) et recuit à $900{ }^{\circ} \mathrm{C}$ pendant 20 min [18] ne montrent qu'une très légère augmentation de la concentration de $\mathrm{Cr}$ au voisinage de la surface. Comme nos échantillons sont implantés avec une dose d'oxygène 100 fois plus faible, nous pensons que le chrome résiduel, qui peut être présent dans nos échantillons, ne peut produire la compensation que nous avons observée.

Le carbone est aussi un accepteur dans GaAs ; sa concentration résiduelle est souvent supérieure à $10^{15} \mathrm{~cm}^{-3}[19,20]$. Makita et al. [21] ont co-implanté dans GaAs de l'oxygène et du carbone de telle manière que leur concentration soit uniforme dans la région implantée. Ils ont déduit des spectres de photoluminescence d'échantillons recuits à différentes températures, que l'oxygène et le carbone forment un complexe mais seulement dans les échantillons recuits à $T<600^{\circ} \mathrm{C}$. Dans nos échantillons recuits à $T>600{ }^{\circ} \mathrm{C}$, le carbone ne devrait plus former de complexes avec l'oxygène. Il est toujours accepteur ; cependant il est difficile d'apprécier son rôle dans la compensation que nous avons observée.

Considérons maintenant l'influence des atomes du réseau GaAs déplacés par le choc frontal des ions implantés; les atomes d'arsenic se retrouvent en excès dans le cristal à une profondeur légèrement plus grande que celle du maximum de la répartition des ions implantés [22]. En extrapolant la concentration des atomes d'arsenic en excès, calculée par Christel et al. [22], pour une implantation d'ions silicium d'énergie $150 \mathrm{keV}$ à une dose de $10^{15} \mathrm{~cm}^{-2}$, on peut estimer très grossièrement que la concentration maximale des atomes d'arsenic en excès est de l'ordre de $10^{13} \mathrm{~cm}^{-3}$, compte tenu que nos échantillons sont multi-implantés en oxygène. D'autre part :

- les concentrations des lacunes d'arsenic électriquement neutres, déterminées par la mesure des durées de vie des positons, varient de $5 \times 10^{16}$ à $5 \times 10^{18} \mathrm{~cm}^{-3}$ [23] dans un lingot de $\mathrm{GaAs}$; elles sont donc nettement plus grandes que les concentrations des atomes d'arsenic en excès produites par l'implantation,
- la compensation de GaAs produite par l'arsenic implanté à une énergie de $900 \mathrm{keV}$ avec une dose de $1 \times 10^{13} \mathrm{~cm}^{-2}$ dans un échantillon recuit à $850{ }^{\circ} \mathrm{C}$, reste localisée dans la région implantée [24].

Tous ces résultats nous amènent à penser que les atomes d'arsenic déplacés par les ions implantés ne produisent pas la compensation observée.

Le gallium étant un élément plus léger que l'arsenic, sa concentration en excès se trouve à une profondeur plus grande que celle de l'arsenic [22] ; d'autre part, les atomes de gallium interstitiels se recombinent rapidement avec les lacunes de gallium [25]. Le gallium déplacé lors de l'implantation ne semble pas expliquer la compensation décrite.

Ainsi les déplacements des atomes du réseau de GaAs provoqués par les différentes implantations ne peuvent être invoqués pour expliquer la compensation observée.

Comparons nos résultats à ceux des échantillons implantés au bore. En effet, l'implantation de bore dans GaAs produit une couche compensée de stabilité thermique satisfaisante [26-28]. Par rapport à celle de nos échantillons, cette couche compensée présente une différence importante : elle reste sensiblement localisée dans la région implantée. Ce résultat est dû probablement à ce que le bore diffuse très peu dans GaAs implanté et recuit à $T \leqslant 900^{\circ} \mathrm{C}$ [29]. Dans le cas de nos échantillons implantés en oxygène et co-implantés en oxygène et en silicium, la compensation s'étend bien au-delà de la région implantée. L'hypothèse d'une diffusion de l'oxygène, lors du recuit, peut être envisagée. Cependant, en raison de la concentration trop faible de cet élément, les mesures de SIMS pour déterminer cette concentration en profondeur dans nos échantillons n'ont pu être réalisées. En effet, la concentration maximale théorique de l'oxygène implanté dans nos échantillons est inférieure à $1,35 \times 10^{16} \mathrm{~cm}^{-3}$. Or la résolution des mesures de SIMS est de l'ordre de $10^{16}-10^{17} \mathrm{~cm}^{-3}[18,30]$. Signalons que Deveaud et al. [18] ont montré que l'oxygène diffuse dans GaAs pendant un recuit de $20 \mathrm{~min}$ à $900^{\circ} \mathrm{C}$ lorsque la dose de l'oxygène implanté est inférieure à $10^{14} \mathrm{~cm}^{-2}$. Lors de la préparation de trois couches successives de GaAs sur un substrat de GaAs par la méthode L.P.E. (Liquid Phase Epitaxy) à partir du Ga fondu et saturé de $\mathrm{O}^{18}$, Chang et al. [31] ont seulement observé, à partir de résultats des mesures de SIMS, une diffusion de $\mathrm{O}^{18}$ de la première couche, préparée à la température la plus élevée $\left(840^{\circ} \mathrm{C}\right)$, vers le substrat. Ces résultats conduisent à penser que, dans notre cas, la diffusion d'oxygène n'a lieu que dans les échantillons recuits à $900^{\circ} \mathrm{C}$. Cette supposition n'est pas en contradiction avec les résultats de Favennec [8] qui a montré que la compensation de GaAs par l'oxygène implanté (énergie : $1,8 \mathrm{MeV}$ et dose : $10^{12} \mathrm{~cm}^{-2}$ ) reste localisée dans la région implantée après un recuit à $700-800{ }^{\circ} \mathrm{C}$. 
Dans le cas des échantillons recuits à $650^{\circ} \mathrm{C}$, la compensation s'étend également au-delà de la région implantée. Comme l'oxygène ne semble pas diffuser à cette température, la compensation est produite vraisemblablement par d'autres types de défauts dont la diffusion est favorisée par la présence de cet oxygène implanté. Rappelons que nous avons déduit des variations de la concentration électrique en fonction de la température, que certains de ces défauts ont une énergie d'ionisation se trouvant dans l'intervalle $0,05-0,11 \mathrm{eV}$. Notons que des défauts intrinsèques $E_{1}$ et $E_{2}$ d'énergies d'ionisation voisines $\left(E_{\mathrm{C}}-E_{\mathrm{t}}=0,045\right.$ et $\left.0,14 \mathrm{eV}\right)$ ont été mis en évidence dans des échantillons de GaAs irradiés par des électrons d'énergie $1 \mathrm{MeV}$ par Pons et al. [32, 33]. Selon ces auteurs et selon Bourgoin et al. [34], ces énergies correspondent respectivement aux changements d'état d'ionisation $\left(2^{-} / /^{-}\right)$et $(-/ 0)$ de la lacune d'arsenic. La concentration de ces lacunes déterminée par annihilation de positons (35-36) est relativement importante. Les défauts présents dans les échantillons implantés en oxygène ou co-implantés en oxygène et en silicium sont certainement plus complexes que les défauts intrinsèques précédents, mais il est vraisemblable que tous ces défauts coexistent. Par la méthode DDLTS (Partie II) nous avons caractérisé dans certains échantillons recuits à $650^{\circ} \mathrm{C}$ ou à $900^{\circ} \mathrm{C}$ un défaut noté $A_{1}$ ayant une énergie d'activation du taux d'émission électronique $E_{\text {a }}$ de $0,11 \mathrm{eV}$. Cette valeur est comparable à celle déterminée précédemment à partir des variations de la concentration électronique en fonction de la température. Cependant la concentration de ce défaut $\mathrm{A}_{1}$ est faible (inférieure à $8 \times 10^{13} \mathrm{~cm}^{-3}$ ) ; son rôle dans la compensation est difficile à apprécier. Les autres défauts mis en évidence également lors de cette étude ont tous des valeurs de $E_{\mathrm{a}}>0,14 \mathrm{eV}$. En supposant négligeable l'énergie d'activation de la section efficace de capture des électrons devant $E_{\mathrm{a}}$, nous avons $E_{\mathrm{a}}=E_{\mathrm{C}}-E_{\mathrm{t}}\left(E_{\mathrm{t}}\right.$ est le niveau d'énergie du défaut). Comme $E_{\mathrm{C}}-E_{\mathrm{t}}>0,14>$ $E_{\mathrm{C}}-E_{\mathrm{F}}$ à toutes les températures de mesures, ces défauts gardent un degré d'ionisation constant. Ils n'interviennent donc pas dans la compensation.

Par ailleurs, nous avons déduit des concentrations électroniques de nos échantillons, implantés à faibles doses, que e nom re e ec $r$

plus grand que le nombre d'atomes d'oxygène implanté. Gecim et al. [37] ont observé le même phénomène. Ces auteurs donnent une diminution moyenne de six électrons par atome d'oxygène implanté dans le cas des faibles doses. Ce résultat montre que l'oxygène ne provoque pas seul la compensation et que celle-ci est probablement produite par l'oxygène associé à différents types de défauts présents dans l'échantillon.

En ce qui concerne les échantillons implantés en oxygène et recuits à $900^{\circ} \mathrm{C}$, la compensation apparaît quand la concentration de l'oxygène implanté est du même ordre de grandeur que la concentration électronique du substrat. Elle augmente avec la dose d'oxygène. En présence de silicium co-implanté, cette compensation devient moins importante. Itoh et al. [38] ont observé des résultats semblables dans le cas des échantillons de GaAs dopé au silicium $\left(n=5 \times 10^{17} \mathrm{~cm}^{-3}\right)$ et implanté en oxygène ; avec une faible dose d'oxygène $\left(n_{\mathrm{O}}=4,42 \times 10^{17} \mathrm{~cm}^{-3}\right)$ l'échantillon est peu compensé tandis qu'avec une forte dose d'oxygène $\left(n_{\mathrm{O}} \sim 8,9 \times 10^{19} \mathrm{~cm}^{-3}\right)$ la compensation est importante et s'étend comme dans notre cas, profondément dans le substrat, au-delà de la région implantée. Gecim et al. [37] ont également mis en évidence des résultats analogues.

D'autre part, dans les échantillons recuits à $900^{\circ} \mathrm{C}$, les concentrations électroniques sont indépendantes de la température de mesure contrairement à celles des échantillons recuits à $650^{\circ} \mathrm{C}$; les défauts d'énergie d'ionisation $0,05-0,11 \mathrm{eV}$ dont nous avons parlé précédemment et qui sont responsables des variations de la concentration électronique en fonction de la température semblent être pratiquement "guéris » par le recuit à $900^{\circ} \mathrm{C}$.

Signalons que l'implantation d'oxygène dans un échantillon de $\mathrm{GaAs}$ du type $\mathrm{p}$ provoque également une compensation [39]. Dans ce cas l'oxygène se comporte comme un donneur tandis que dans GaAs du type $n$, il se comporte comme un accepteur.

En résumé, la compensation observée dans $\mathrm{GaAs}$ implanté en oxygène dépend de nombreux paramètres qui sont, pour la plupart d'entre eux, mal connus, ce qui rend très difficile son interprétation.

\section{Conclusion.}

Cette étude nous a permis de tirer les conclusions suivantes :

- la compensation de GaAs du type $n$ par implantation d'oxygène n'apparaît que lorsque la concentration de cet élément est du même ordre de grandeur que la concentration électronique du substrat. Elle augmente avec la dose d'oxygène implanté ;

- une co-implantation d'oxygène et de silicium produit une compensation plus importante du GaAs

est effectué à $650^{\circ} \mathrm{C}$. Il semble qu'il existe une certaine interaction entre l'oxygène et le silicium implantés ;

- la compensation produite s'étend au-delà de la région implantée. Lorsque la température du recuit est de $650^{\circ} \mathrm{C}$, la compensation est due en partie à des défauts, dont l'énergie d'ionisation est comprise entre 0,05 et $0,11 \mathrm{eV}$ au-dessous de la bande de conduction ;

- dans les échantillons recuits à $900^{\circ} \mathrm{C}$, la compensation reste importante dans le cas d'une 
implantation en oxygène seul mais devient plus faible dans le cas d'une co-implantation $\mathrm{Si}+\mathrm{O}$. Le silicium se comporte en donneur. La concentration électronique est indépendante de la température de mesure. Un recuit à $900^{\circ} \mathrm{C}$ semble donc nécessaire pour « guérir » les défauts créés par l'implantation;

- dans nos échantillons implantés ou co-implantés, le nombre de donneurs compensés est nettement plus grand que le nombre d'atomes d'oxygène implantés ; ceci met en évidence que l'oxygène ne produit pas seul la compensation;

- la compensation ne peut être justifiée par une diffusion des impuretés résiduelles $(\mathrm{Cr}$, et $\mathrm{C}$ accepteurs dans $\mathrm{GaAs}$ ) du substrat vers la surface.
L'interprétation du rôle du $\mathrm{Si}$ comme celui de l'oxygène, dans la compensation de GaAs que nous avons observée, est difficile, car il y a dans ce semiconducteur un nombre relativement important d'impuretés résiduelles dont la concentration est de l'ordre de $10^{15} \mathrm{~cm}^{-3}$. Néanmoins nous pensons que la présence de l'oxygène et du silicium implantés doit modifier l'équilibre physico-chimique entre les défauts présents dans le substrat. Cette modification doit probablement entraîner la formation d'autres défauts plus complexes qui produisent la compensation. L'interprétation précise du mécanisme de ce phénomène de compensation nécessite encore d'autres études.

\section{Bibliographie}

[1] Hilsum C., Rose-InNes A. C., Semiconducting IIIV Compounds (Oxford, Pergamon) 1961, p. 142.

[2] Ainslie N. G., Bliens S. E., Wood J. F., J. Appl. Phys. 33 (1962) 2391.

[3] Fertin J. L., Lebailly J. L., Deyris E., Inst. Phys. Conf. Ser. 3, Gallium Arsenide (1967) p. 46.

[4] Woodall J. M., Woods J. F., Solid State Commun. 4 (1966) 33-36.

[5] Woodall J. M., Trans. Metall. Soc. 239 (1967) 378.

[6] Martin G. M., Jacob G., Hallais J. P., Grainger F., Roberts J. A., ClegG B., Blood P., Poiblaud G., J. Phys. C 15 (1982) 1841.

[7] Favennec P. N., Pelous G., Binet M., Baudet $\mathrm{P}$., Ion implantation in semiconductors (Plenum Press) 1974, p. 621.

[8] Favennec P. N., J. Appl. Phys. 47 (1976) 2532.

[9] Weiner M. E., Jordan A. S., J. Appl. Phys. 43 (1972) 1767.

[10] Partie II. Etude des défauts profonds (dans ce même numéro).

[11] Le Bloa A., Dang Tran Quan, Favennec P. N., Revue Phys. Appl. 21 (1986) 489.

[12] Shikano K., Kobayashi K., Favennec P. N., Appl. Phys. Lett. 46 (1985) 391.

[13] Abdalla, Palmier J. F., Desfeux C., Inst. Phys. Conf. Ser. $N^{\circ} 24$ (1975) chapitre VI, p. 342.

[14] Berth M., Venger C., Martin G. M., Electron. Lett. 17 (1981) 873.

[15] Martin G. M., Makram-Ebeid S., Ngo Tich Phuoc, Berth M., Wenger C., Proceeding of the 2nd Conference on Semi-Insulating III-V Materials (Evian, France) April 1982.

[16] Zohta Y., Appl. Phys. Lett. 17 (1970) 1277.

[17] Yeo Y. K. and Hengehold R. L., YoNG YUN KIM, Ezis A., Park Y.S., Ehret J. E., J. Appl. Phys. 58 (1985) 4083.

[18] Deveaud B., Favennec P. N., L'Haridon H., Armand C., Martinez A., Conf. on semiinsulating III-V Materials (Evian, France 1982) Eds. S. Makram-Ebeid, T. Tuck (Shiva Publ. Ltd, U.K.).

[19] Lagowski J., Lin D. G., Chen T. P., Skovoronski M., Gatos H. C., Appl. Phys. Lett. 47 (1985) 929.
[20] Look D. C., Walters D. C., Meyer J. R., Solid State Commun. 42 (1982) 745.

[21] Makita Y., Appl. Phys. Lett. 47 (1985) 623.

[22] Christel L. A., Gibbons J. F., J. Appl. Phys. 52 (1981) 5051.

[23] Dlubeck D., Brunmer O., Plazaola P., HautoJARVI, J. Phys. C 19 (1986) 331.

[24] Pons D., Bourgoin J. C., J. Phys. C 18 (1985) 3839.

[25] Rao E. V. K., Duhamel N., Favennec P. N., L'Haridon H., Appl. Phys. 40 (1982) 960.

[26] Toulouse B., Favennec P. N., GuivarC'H A., Pelous G., Inst. Phys. Conf. Ser. 45 (1979) 501.

[27] Toulouse B., Thèse Université de Rennes (1979).

[28] Martin G., Secordel P., Venger C., J. Appl. Phys. 53 (1982) 8706.

[29] Hopkins C. G., Deline V. R., Blattner R. J., Evans C. A., J. R., Maggee T. J., Appl. Phys. Lett. 37 (1980) 635.

[30] Lagowsi J., Lin D. G., Adyama T., Gatos H. C., Appl. Phys. Lett. 44 (1984) 336.

[31] Chang S. S. C., Kisker D. W., Stevenson D., Solid State Electron. 28 (1985) 479.

[32] Pons D., Mooney P. M., Bourgoin J. C., J. Appl. Phys. 51 (1980) 2038.

[33] Pons D., Bourgoin J. C., J. Phys. C 18 (1985) 3839.

[34] Bourgoin J. C., Von Bardeleben H. J., STIEVeNART D., J. Appl. Phys. 64 (1988) R65.

[35] Dlubeck D., Brumer O., Plazaola P., HotoJARVi P., J. Phys. C 19 (1986) 331.

[36] Dannefaer S., Kerr D., J. Appl. Phys. 60 (1986) 591.

[37] Gecim S., Sealy B., Stephens K. G., Electr. Lett. 14 (1978) 306.

[38] Iтон T., Tsuchiya T., Takeuchi M., Jpn J. Appl. Phys. 15 (1976) 2277.

[39] Humer-Hager T., Zwicknagl P., Appl. Phys. Lett. 52 (1988) 63.

[40] Lagowski J., Gatos H. C., Parsey J. M., Wada K., Kaminska M., Walukiewicz W., Appl. Phys. Lett. 40 (1982).

[41] Weber E. R., EnNen H., Kaufmann V., WindsCHEIFF J., SCHNEIDER J., WASINSKI T., J. Appl. Phys. 53 (1982) 6140. 